\title{
A CHARACTERIZATION OF THE INVARIANT MEASURES FOR AN INFINITE PARTICLE SYSTEM WITH INTERACTIONS( ${ }^{(1)}$
}

\author{
BY \\ THOMAS M. LIGGETT
}

\begin{abstract}
Let $p(x, y)$ be the transition function for a symmetric, irreducible, transient Markov chain on the countable set $S$. Let $\eta_{t}$ be the infinite particle system on $S$ with the simple exclusion interaction and one-particle motion determined by $p$. A characterization is obtained of all the invariant measures for $\eta_{t}$ in terms of the bounded functions on $S$ which are harmonic with respect to $p(x, y)$. Ergodic theorems are proved concerning the convergence of the system to an invariant measure.
\end{abstract}

1. Introduction. Spitzer's motivation for introducing his models of infinite particle systems with interactions came, to a large extent, from the fact that many of them have interesting classes of invariant measures [8]. The classical Gibbs measures of statistical mechanics, for example, are invariant for one of his most interesting models - that of speed change and exclusion. Much of the study of invariant measures for these models has concentrated on showing that certain measures, which are more or less explicitly known, are stationary for the model in question. This raises the natural problem of finding all of the invariant measures in any given case. In this paper, we solve this problem for one of Spitzer's simplest models-that of simple exclusion.

The simple exclusion model is described in terms of a transition function $p(x, y)$ for a Markov chain on a countable set $S$. If there were no interactions, the particles in the system would move independently of each other according to a continuous time Markov chain on $S$ with transition probabilities $p(x, y)$ and waiting times with constant parameter 1 at each point. The interaction of simple exclusion is superimposed on this motion in a way which makes it impossible for more than one particle to be at any point in $S$ at any given time. If a particle attempts a transition to a point which is already occupied, then that transition is prohibited, and the particle must remain where it is until it next attempts a transition.

Since multiple occupancy is excluded in this model, the set of all configurations is $X=\{0,1\}^{S}$, which is given the product topology. The interpretation of $\eta \in X$

Received by the editors July 7, 1972.

AMS (MOS) subject classifications (1970). Primary 60K35; Secondary 47A35.

Key words and phrases. Infinite particle systems, invariant measures, ergodic theorems.

(') The preparation of this paper was supported in part by NSF Grant GP-28258 and in part by the Air Force Office of Scientific Research, Air Force Systems Command, USAF, under Grant AFOSR 69-1781. The United States Government is authorized to reproduce and distribute reprints for governmental purposes notwithstanding any copyright notation hereon. 
is that $x \in S$ is occupied if $\eta(x)=1$ and vacant if $\eta(x)=0$. The infinite particle system can then be defined as the Markov process $\eta_{t}$ with state space $X$ whose infinitesimal generator is described in the following way. Let $C(X)$ be the space of all continuous functions on $X$ with the supremum norm, and let $\exists \subset C(X)$ be the subset of those functions which depend on only finitely many coordinates. For $x, y \in S$ and $\eta \in X$ so that $\eta(x)=1$ and $\eta(y)=0$, let $\eta_{x, y}$ denote the configuration which results from $\eta$ after a transition has occurred from $x$ to $y$ :

$$
\begin{aligned}
\eta_{x, y}(u) & =\eta(u) & & \text { if } u \neq x, y, \\
& =1 & & \text { if } u=y, \\
& =0 & & \text { if } u=x .
\end{aligned}
$$

For $f \in \mathcal{G}$, define $\Omega f \in C(X)$ by

$$
\Omega f(\eta)=\sum_{\eta(x)=1 ; \eta(y)=0} p(x, y)\left[f\left(\eta_{x, y}\right)-f(\eta)\right] .
$$

The generator of the process $\eta_{t}$ is then the closure of $\Omega$ in $C(X)$. It is shown in [6] that $\Omega$ has a unique closure which is the generator of a strongly continuous semigroup $S(t)$ of contractions on $C(X)$, and therefore generates a strong Markov process on $X$, provided that $p(x, y)$ satisfies $\sup _{y} \sum_{x} p(x, y)<\infty$. The results of this paper will be restricted to the case in which $p(x, y)$ is symmetric, in which case this condition is automatically satisfied. The semigroup $S(t)$ on $C(X)$ induces in the usual way a semigroup on the space of probability measures on $X$ via the relation

$$
\int f(\eta)[S(t) \mu](d \eta)=\int[S(t) f](\eta) \mu(d \eta) .
$$

Spitzer observed $\left[8\right.$, p. 280] that if $p(x, y)$ is symmetric, then $\eta_{t}$ possesses the following important property, which plays a fundamental role in our work and explains one of the basic reasons for the symmetry requirement:

Theorem 1.1 (Spitzer). Suppose $p(x, y)$ is a symmetric function of $x$ and $y$. If $\zeta_{1}, \zeta_{2} \in X$ and $\Sigma_{x} \zeta_{2}(x)<\infty$, then

$$
P^{\zeta_{1}}\left(\eta_{t} \geq \zeta_{2}\right)=P^{\zeta_{2}}\left(\zeta_{1} \geq \eta_{t}\right),
$$

where the inequalities are to be interpreted componentwise.

This result of ten permits the reduction of a problem concerning the infinite particle system to one relating only to the corresponding finite particle system. For example, an immediate consequence of this theorem which shows its relevance to the invariant measure problem is

Corollary 1.2. Suppose $p(x, y)$ is symmetric. If $\mu$ is a probability measure on $X$, define a function $g$ on $X$ by $g(\eta)=\mu\{\zeta \mid \zeta \geq \eta\}$. Then $\mu$ is invariant for the process $\eta_{t}$ if and only if $E^{\eta}\left[g\left(\eta_{t}\right)\right]=g(\eta)$ for all $\eta$ for which $\sum_{x} \eta(x)<\infty$. 
Proof. By definition, $\mu$ is invariant if and only if $P^{\mu}\left\{\eta_{t} \geq \eta\right\}=g(\eta)$ for all $\eta$ for which $\sum_{x} \eta(x)<\infty$. But, for such $\eta$,

$$
\begin{aligned}
P^{\mu}\left\{\eta_{t} \geq \eta\right\} & =\int P^{\zeta}\left(\eta_{t} \geq \eta\right) \mu(d \zeta)=\int P^{\eta}\left(\eta_{t} \leq \zeta\right) \mu(d \zeta) \\
& =\int\left[\sum_{\gamma \leq \zeta} P^{\eta}\left(\eta_{t}=\gamma\right)\right] \mu(d \zeta) \\
& =\sum_{\gamma} P^{\eta}\left(\eta_{t}=\gamma\right) g(\gamma)=E^{\eta}\left[g\left(\eta_{t}\right)\right] .
\end{aligned}
$$

It follows from this that any probability measure on $X$ with respect to which the random variables $\eta(x)$ are exchangeable is invariant for $\left\{\eta_{t}\right\}$, since in that case $g(\eta)$ depends only on $\Sigma_{x} \eta(x)$. Our basic problem then is to find necessary and sufficient conditions on $p(x, y)$ so that there are no other invariant measures for $\left\{\eta_{t}\right\}$, and to find all other invariant measures in cases where others exist.

We solve this problem under the assumptions that $p(x, y)$ is a symmetric function of $x$ and $y$ and that the Markov chain on $S$ with transition probabilities $p(x, y)$ is irreducible and transient. These assumptions will be made throughout the remainder of this paper (except in $\$ 2$ ). In order to state the main results, the following notation is needed. $I$ will denote the set of all probability measures on $X$ which are invariant for the process $\left\{\eta_{t}\right\}$, and $\mathscr{J}_{e}$ will be the set of extreme points of $\mathscr{I}$. Since $\mathscr{I}$ is a compact and convex set in the topology of weak convergence of measures, the Krein-Millman theorem guarantees that $I$ is the closed convex hull of $g_{e}$. Put

$$
\mathcal{H}=\left\{\alpha(\cdot) \text { on } S \mid 0 \leq \alpha(x) \leq 1 \text { and } \sum_{y} p(x, y) \alpha(y)=\alpha(x) \text { for all } x \in S\right\}
$$

Then $\&$ contains every constant between zero and one, since $\sum_{y} p(x, y)=1$ for each $x$. Finally, define $\nu_{\alpha}$ for $\alpha \in \mathcal{A}$ to be the product measure on $X$ such that

$$
\nu_{\alpha}\{\eta \mid \eta \geq \gamma\}=\prod_{\gamma(x)=1} \alpha(x)
$$

for all $\gamma \in X$ such that $\sum_{x} \gamma(x)<\infty$. The main characterization theorem is then

Theorem 1.3. Assume that $p(x, y)$ is the transition function for a symmetric, irreducible, transient Markov chain on $S$. Then

(a) $\mu_{\alpha}=\lim _{t \rightarrow \infty} S(t) \nu_{\alpha}$ exists for each $\alpha \in \mathcal{H}$, and $\mu_{\alpha} \in I_{e}$.

(b) $\mu_{\alpha}\{\eta \mid \eta(x)=1\}=\alpha(x)$ for all $x \in S$.

(c) The map $\alpha \rightarrow \mu_{\alpha}$ gives a one-to-one and onto correspondence between $\&$ and $\mathscr{D}_{e}$.

(d) $\mu_{\alpha}=\nu_{\alpha}$ if and only if $\alpha$ is constant.

(e) $\mathscr{I}_{e}=\{\mu \in \mathcal{I} \mid \mu\{\eta \mid \eta(x)=1, \eta(y)=1\} \leq \mu\{\eta \mid \eta(x)=1\} \mu\{\eta \mid \eta(y)=1\}$ for all $x \neq y\}$. 
Corollary 1.4. I consists only of the measures with respect to which the random variables $\eta(x)$ are exchangeable if and only if the only bounded solutions of $\sum_{y} p(x, y) \alpha(y)=\alpha(x)$ are the constants. In particular, this is true if $S$ is an Abelian group and $p(x, y)=p(0, y-x)$.

Proof. The first statement is a consequence of De Finetti's theorem [2, p. 225], and the second follows from the first by the Choquet-Deny theorem [1].

Remark. There are many choices for $p(\cdot, \cdot)$ which satisfy the assumptions of the theorem and for which $\&$ does not consist entirely of constants, and hence $I_{e}$ does not consist entirely of product measures. An example is the simple random walk on the free group on two generators.

Theorem 1.3 will be proved in $\S 4$. We conjecture that the theorem is true without the assumption of transience, although the proof in the recurrent case will probably be quite different. (In the recurrent case, of course, \& consists only of constants.) The following remarks should be helpful in understanding the role of the transience assumption in the proof. Theorem 1.1 and its corollary permit the reduction of the problem to one dealing with the system consisting only of a finite number of particles. Since an interaction occurs only if a particle attempts a transition to an occupied site, one would expect that if $p(x, y)$ were transient, then only a finite number of interactions would occur over all time for the finite particle system. Therefore one would expect the finite particle interacting system to behave very much like the corresponding finite particle system without interactions, which is much easier to study. The relationship between the interacting and noninteracting finite particle systems is studied in $\S 3$. In doing this, several results concerning Markov chains are needed, and these are proved in \$2. Added in proof: Theorem 1.3 does hold in the recurrent case. See forthcoming papers by Spitzer and Liggett in Trans. Amer. Math. Soc.

The final section deals with ergodic theorems for the process $\eta_{t}$. Since the system has infinitely many invariant measures, they take the form of describing the domain of attraction of each of these. It suffices to do this for extreme invariant measures, so the problem is to describe $\left\{\mu\right.$ on $\left.X \mid S(t) \mu \rightarrow \mu_{\alpha}\right\}$ for $\alpha \in \mathcal{H}$ in some way which is independent of the infinite particle system. In order to state the main result, put

$$
p_{t}(x, y)=e^{-t} \sum_{k=0}^{\infty} \frac{t^{k}}{k !} p^{(k)}(x, y)
$$

where $p^{(k)}(x, y)$ are the $k$-step transition probabilities for the Markov chain on $S$ which has transition function $p(x, y)$.

Theorem 1.5. Suppose the conditions of Theorem 1.3 hold, that $\mu$ is a probability measure on $X$, and that $\alpha \in \mathcal{H}$. Then $S(t) \mu \rightarrow \mu_{\alpha}$ as $t \rightarrow \infty$ if and only if

$$
\sum_{y} p_{t}(x, y)[\eta(y)-\alpha(y)] \rightarrow 0
$$


in probability with respect to $\mu$ as $t \rightarrow \infty$ for each $x \in S$.

Remark. Intuitively, the content of condition (1.6) is that the random variables $\eta(x)$ satisfy a type of mixing condition with respect to $\mu$. This will be seen more explicitly in Corollary 5.5.

As a consequence of this theorem, it will be shown in $\S 5$ that if $S$ is an Abelian group, $p$ satisfies $p(x, y)=p(0, y-x)$ in addition to the conditions of Theorem 1.3 , and $\mu$ is stationary and ergodic, then $S(t) \mu \rightarrow \mu_{\alpha}$ as $t \rightarrow \infty$, where $\alpha$ is the constant $\mu\{\eta \mid \eta(x)=1\}$. In particular, this holds in case $p(x, y)$ is the transition function for the simple random walk in $Z^{k}$ for $k \geq 3$. Using entirely different techniques, Spitzer (private communication) has proved this result in this case for all $k \geq 1$.

Results of this type for other models of infinite particle systems with interactions have been obtained by Holley (see [3], [4], and [5]).

2. Preliminary results. This section is devoted to obtaining several results concerning discrete time Markov chains which will be needed in the next section. They will be applied there to show that the finite particle interacting process is absolutely continuous with respect to the finite particle noninteracting process.

Lemma 2.1. Let $\mu$ and $\nu$ be probability measures on a measurable space $(\Omega, \mathcal{B})$, and let $\mathcal{B}_{n}$ be an increasing sequence of sub- $\sigma$-algebras whose union generates $\mathcal{B}$. Let $\mu_{n}$ and $\nu_{n}$ be the restrictions of $\mu$ and $\nu$ to $\mathcal{B}_{n}$ respectively. Suppose that $\nu_{n} \ll \mu_{n}$ for each $n$, and that $f=\lim _{n \rightarrow \infty} d \nu_{n} / d \mu_{n}$ exists and is finite a.s., with respect to $\nu$. Then $\nu \ll \mu$.

Proof. For each $n$, let $\mu_{n}=\mu_{n}^{\text {a.c. }}+\mu_{n}^{\text {s. }}$ be the Lebesgue decomposition of $\mu_{n}$ relative to $\nu_{n}$. Then

$$
d \mu_{n}^{\text {a.c. }} / d \nu_{n}=\left[d \nu_{n} / d \mu_{n}\right]^{-1}
$$

So, for $A \in \mathcal{B}_{n}$,

$$
\mu_{n}(A) \geq \mu_{n}^{\text {a.c. }}(A)=\int_{A}\left[\frac{d \nu_{n}}{d \mu_{n}}\right]^{-1} d \nu_{n},
$$

and therefore

$$
\mu(A) \geq \int_{A}\left[\frac{d \nu_{n}}{d \mu_{n}}\right]^{-1} d \nu
$$

By Fatou's lemma,

$$
\mu(A) \geq \int_{A} f^{-1} d \nu
$$

Since $\cup_{n} \mathcal{B}_{n}$ generates $\mathcal{B},(2.2)$ holds for all $A \in \mathcal{B}$. Since $f^{-1}>0$ a.s. with respect to $\nu$, it follows that $\nu \ll \mu$. 
This will now be applied to Markov chains. Let $p(x, y)$ be the transition probabilities for a Markov chain $X_{n}$ on the countable set $S$. Take $C \subset S$, and let $Y_{n}$ be the Markov chain on $C$ with transition probabilities

$$
\begin{aligned}
q(x, y) & =p(x, y) / s(x) & & \text { if } y \in C, \\
& =0 & & \text { if } y \notin C,
\end{aligned}
$$

where $s(x)=\sum_{y \in C} p(x, y)$. (If $s(x)=0$, put $q(x, x)=1$.) Fix a common starting point $x_{0} \in C$ for both chains, and let $\mu$ and $\nu$ be the measures on $S^{\infty}$ generated by $\left\{X_{n}\right\}$ and $\left\{Y_{n}\right\}$ respectively.

Theorem 2.3. If $\sum_{n}\left[1-s\left(Y_{n}\right)\right]<\infty \quad$ a.s., then $\nu \ll \mu$.

Proof. First note that by assumption $\nu\left\{\vec{x} \in S^{\infty} \mid s\left(x_{i}\right)=0\right.$ for some $\left.i\right\}=0$. Therefore, we may assume throughout that $s(x)>0$ for all $x \in C$. Let $\mathcal{B}_{n}$ be the $\sigma$-algebra generated by the first $n$ coordinates, and $\mu_{n}$ and $\nu_{n}$ be the restrictions of $\mu$ and $\nu$ to $\mathcal{B}_{n}$. Then $\nu_{n} \ll \mu_{n}$ and $d \nu_{n} / d \mu_{n}$ is the function on $S^{n}$ given by

$$
\frac{d \nu_{n}}{d \mu_{n}}\left(x_{1}, \ldots, x_{n}\right)=\frac{q\left(x_{0}, x_{1}\right) \cdots q\left(x_{n-1}, x_{n}\right)}{p\left(x_{0}, x_{1}\right) \cdots p\left(x_{n-1}, x_{n}\right)}
$$

if $x_{i} \in C$ for each $i \leq n$ and the denominator is not zero, and $d \nu_{n} / d \mu_{n}=0$ otherwise. So, a version of $d \nu_{n} / d \mu_{n}$ is

$$
\begin{aligned}
\frac{d \nu_{n}}{d \mu_{n}}\left(x_{1}, \ldots, x_{n}\right) & =\prod_{i=0}^{n-1} \frac{1}{s\left(x_{i}\right)} & & \text { if } x_{i} \in C \text { for all } i, \\
& =0 & & \text { otherwise. }
\end{aligned}
$$

But by assumption, $\sum_{n}\left[1-s\left(x_{n}\right)\right]<\infty$ a.s. with respect to $\nu$, so $\prod_{n=0}^{\infty} s\left(x_{n}\right)$ $>0$ a.s. with respect to $\nu$. The desired conclusion then follows from Lemma 2.1.

Remark. It is interesting to note that the assumption of this theorem can be interpreted as saying that the number of times that $Y_{n}$ "attempts" to leave $C$ is finite.

For the final result in this section, we assume that the Markov chain with transition probabilities $p(x, y)$ is transient, and define

$$
G(x, y)=\sum_{k=0}^{\infty} p^{(k)}(x, y) .
$$

Lemma 2.5. For $x \neq y, G(x, x)+G(y, y)-G(x, y)-G(y, x) \geq 1$.

Proof. For $x \in S, \tau_{x}$ will denote the hitting time of $x$. Then $G(x, x)$ $=\left[P^{x}\left(\tau_{x}=\infty\right)\right]^{-1}$, and $G(x, y)=P^{x}\left(\tau_{y}<\infty\right) G(y, y)$ for $x \neq y$. Also,

$$
P^{x}\left(\tau_{x}=\infty\right) \leq P^{x}\left(\tau_{y}=\infty\right)+P^{y}\left(\tau_{x}=\infty\right)
$$


follows from $P^{x}\left(\tau_{x}<\infty\right) \geq P^{x}\left(\tau_{y}<\infty\right) P^{y}\left(\tau_{x}<\infty\right)$. Therefore, for $x \neq y$,

$$
\begin{aligned}
G(x, x) & +G(y, y)-G(x, y)-G(y, x) \\
& =P^{y}\left(\tau_{x}=\infty\right) G(x, x)+P^{x}\left(\tau_{y}=\infty\right) G(y, y) \geq 1
\end{aligned}
$$

by (2.6).

3. The finite particle system. While the infinite particle system with the simple exclusion interaction must be discussed in continuous time, it is convenient in discussing the system with only finitely many particles to consider the embedded discrete time process. This is then a Markov chain $Y_{k}$ on $T_{n}=\left\{\vec{x} \in S^{n} \mid x_{i} \neq x_{j}\right.$ for $i \neq j\}$, where $n$ is the number of particles in the system. It is defined on all of $S^{n}$ by letting all points in $S^{n} \backslash T_{n}$ be absorbing. Its transition operator $V_{n}$ is then defined for nonnegative functions $f$ on $S^{n}$ by

$$
\begin{aligned}
V_{n} f(\vec{x})= & E^{\vec{x}} f\left(Y_{1}\right)=\frac{1}{n}\left[\sum_{\substack{i=1 \\
i \neq j}}^{n} \sum_{j=1}^{n} p\left(x_{i}, x_{j}\right)\right] f(\vec{x}) \\
& +\frac{1}{n} \sum_{i=1}^{n} \sum_{u \neq x_{j} ; j \neq i} p\left(x_{i}, u\right) f\left(x_{1}, \ldots, x_{i-1}, u, x_{i+1}, \ldots, x_{n}\right)
\end{aligned}
$$

if $\vec{x} \in T_{n}$, and $V_{n} f(\vec{x})=f(\vec{x})$ otherwise. The transition operator for the corresponding Markov chain $X_{k}$ on $S^{n}$ in which the particles are permitted to move independently is

$$
U_{n} f(\vec{x})=E^{\vec{x}} f\left(X_{1}\right)=\frac{1}{n} \sum_{i=1}^{n} \sum_{u} p\left(x_{i}, u\right) f\left(x_{1}, \ldots, x_{i-1}, u, x_{i+1}, \ldots, x_{n}\right) .
$$

Much of this section is devoted to the study of the relation between the processes $X_{k}$ and $Y_{k}$, the results of which will be used in the next section to study the general infinite particle system. In order to make the required comparisons, it is necessary to impose throughout the conditions of Theorem 1.3. Since $p(x, y)$ is transient, $G(x, y)$ can be defined as in (2.4). This will be useful in studying the two particle system. For the general $n$ particle system (with $n \geq 2$ ), we need

$$
G_{n}\left(x_{1}, \ldots, x_{n}\right)=\frac{1}{n} \sum_{\substack{i=1 \\ i \neq j}}^{n} \sum_{j=1}^{n} G\left(x_{i}, x_{j}\right) .
$$

The first result states that $G_{n}$ is excessive for the operator $U_{n}$, and in fact that it is the potential of a nonnegative function :rith support on $S^{n} \backslash T_{n}$.

Lemma 3.1. Fix $n \geq 2$, and let

$$
h_{l}(\vec{x})=\frac{1}{n} \sum_{\substack{i=1 \\ i \neq j}}^{n} \sum_{\substack{j=1 \\ i \neq j}}^{n} p^{(l)}\left(x_{i}, x_{j}\right)
$$


for $l=0,1,2, \ldots$, and $\vec{x} \in S^{n}$. Then

$$
\sum_{k=0}^{\infty} U_{n}^{k} h_{l}=\frac{n}{2} \sum_{k=l}^{\infty} h_{k}
$$

In particular, $\sum_{k=0}^{\infty} U_{n}^{k} h_{0}=(n / 2) G_{n}$.

Proof. A simple computation gives $U_{n} h_{l}=(n-2) h_{l} / n+2 h_{l+1} / n$. So, by induction,

$$
U_{n}^{k} h_{l}=\sum_{j=0}^{k}\left(\begin{array}{l}
k \\
j
\end{array}\right)\left(1-\frac{2}{n}\right)^{k-j}\left(\frac{2}{n}\right)^{j} h_{l+j}
$$

Summing on $k$ yields (3.2).

This is now used to show that if the $n$ particles move independently, they tend to get far away from each other.

Lemma 3.3. $G_{n}\left(X_{k}\right) \rightarrow 0$ a.s. as $k \rightarrow \infty$ for any initial configuration $\vec{x} \in S^{n}$ of particles.

Proof. By Lemma 3.1, $U_{n}^{k} G_{n}(\vec{x})=E^{\vec{x}}\left[G_{n}\left(X_{k}\right)\right] \downarrow 0$, so $G_{n}\left(X_{k}\right) \rightarrow 0$ in $L_{1}$. Since $U_{n} G_{n} \leq G_{n}, G_{n}\left(X_{k}\right)$ is a supermartingale, and therefore $G_{n}\left(X_{k}\right) \rightarrow 0$ a.s.

For $n \geq 2$ and $\vec{x} \in T_{n}$, define

$$
g_{n}(\vec{x})=\frac{1}{n} \sum_{\substack{i=1 \\ i \neq j}}^{n} \sum_{\substack{j=1 \\ n}}^{n} p\left(x_{i}, x_{j}\right)
$$

Lemma 3.4. $\sum_{k=0}^{\infty} V_{n}^{k} g_{n} \leq n G_{n}$ for $\vec{x} \in T_{n}$.

Proof. For $\vec{x} \in T_{n}$,

$$
\begin{aligned}
U_{n} G_{n}(\vec{x})-V_{n} G_{n}(\vec{x}) & =\frac{1}{n^{2}} \sum_{i=1}^{n} \sum_{j=1}^{n} p\left(x_{i}, x_{j}\right)\left[G\left(x_{i}, x_{i}\right)+G\left(x_{j}, x_{j}\right)-2 G\left(x_{i}, x_{j}\right)\right] \\
& \geq \frac{1}{n} g_{n}(\vec{x})
\end{aligned}
$$

by Lemma 2.5. By Lemma 3.1, $U_{n} G_{n}(\vec{x})=G_{n}(\vec{x})$ for $\vec{x} \in T_{n}$. Therefore,

$$
g_{n}(\vec{x}) \leq n\left[G_{n}(\vec{x})-V_{n} G_{n}(\vec{x})\right]
$$

Since $V_{n}$ maps functions on $T_{n}$ to functions on $T_{n}, V_{n}$ can be applied repeatedly to (3.5). Summing the resulting inequalities yields the desired conclusion.

Theorem 3.6. $G_{n}\left(Y_{k}\right) \rightarrow 0$ a.s. as $k \rightarrow \infty$ for any initial configuration $\vec{x} \in T_{n}$ of particles. 
Proof. Let $\tilde{Y}_{k}$ be the Markov chain on $T_{n}$ which is obtained from $Y_{k}$ by "ignoring" attempts to go to points in $S^{n} \backslash T_{n}$. In other words, $\tilde{Y}_{k}$ is the Markov chain with transition operator

$$
\tilde{V}_{n} f(\vec{x})=\frac{1}{n} \sum_{i=1}^{n} \sum_{u \neq x_{j}, j \neq i} p\left(x_{i}, u\right) f\left(x_{1}, \ldots, x_{i-1}, u, x_{i+1}, \ldots, x_{n}\right) /\left(1-g_{n}(\vec{x})\right)
$$

Since $\tilde{Y}_{k}$ differs from $Y_{k}$ only by a random change of time and $Y_{k}$ is not eventually constant with positive probability, it suffices to show that $G_{n}\left(\tilde{Y}_{k}\right) \rightarrow 0$ a.s. Let $\mu$ and $\nu$ be the probability measures on $\left(S^{n}\right)^{\infty}$ generated by the Markov chains $X_{k}$ and $\tilde{Y}_{k}$ respectively with the same initial state $\vec{x} \in T_{n}$. By Lemma 3.4, $E\left[\sum_{k=0}^{\infty} g_{n}\left(Y_{k}\right)\right]<\infty$, so $\sum_{k=0}^{\infty} g_{n}\left(Y_{k}\right)<\infty$ a.s. But $\sum_{k=0}^{\infty} g_{n}\left(\tilde{Y}_{k}\right)$ $\leq \sum_{k=0}^{\infty} g_{n}\left(Y_{k}\right)$, since the summands are the same in the two series, but some of the terms are repeated several times on the right-hand side. Therefore $\sum_{k=0}^{\infty} g_{n}\left(\tilde{Y}_{k}\right)<\infty$ a.s. By Theorem 2.3, $\nu \ll \mu$. Let

$$
A=\left\{\left(\vec{x}_{1}, \vec{x}_{2}, \ldots\right) \in\left(S^{n}\right)^{\infty} \mid G_{n}\left(\vec{x}_{k}\right) \rightarrow 0\right\} .
$$

Then $\mu(A)=1$ by Lemma 3.3, so $\nu(A)=1$ also. This shows that $G_{n}\left(\tilde{Y}_{k}\right) \rightarrow 0$ a.s., thus completing the proof.

Corollary 3.7. If $f$ is any bounded function on $T_{n}$ such that $|f| \leq G_{n}$, then $V_{n}{ }^{k} f(\vec{x}) \rightarrow 0$ as $k \rightarrow \infty$.

Proof. If $Y_{k}$ has initial state $\vec{x} \in T_{n}$, then $f\left(Y_{k}\right) \rightarrow 0$ a.s. by Theorem 3.6. Since $f$ is bounded, $V_{n}{ }^{k} f(\vec{x})=E^{\vec{x}}\left[f\left(Y_{k}\right)\right] \rightarrow 0$ as $k \rightarrow \infty$ by the bounded convergence theorem.

The following lemma will be important in relating $X_{k}$ and $Y_{k}$.

Lemma 3.8. If $0 \leq f \leq 1$ on $S^{n}$, then $\left|U_{n}^{k} f(\vec{x})-V_{n}{ }^{k} f(\vec{x})\right| \leq n G_{n}(\vec{x})$ for $\vec{x} \in T_{n}$.

Proof. Write

$$
U_{n}^{k} f-V_{n}^{k} f=\sum_{i=0}^{k-1} V_{n}^{i}\left[U_{n}-V_{n}\right] U_{n}^{k-i-1} f .
$$

A short computation shows that if $0 \leq h \leq 1$ on $S^{n}$, then $\left|U_{n} h(\vec{x})-V_{n} h(\vec{x})\right|$ $\leq g_{n}(\vec{x})$ for $\vec{x} \in T_{n}$. Therefore,

$$
\left|U_{n}^{k} f(\vec{x})-V_{n}{ }^{k} f(\vec{x})\right| \leq \sum_{i=0}^{k-1} V_{n}^{i} g_{n}(\vec{x}) \leq \sum_{i=0}^{\infty} V_{n}^{i} g_{n}(\vec{x}) \leq n G_{n}(\vec{x})
$$

for $\vec{x} \in T_{n}$ by Lemma 3.4 .

For $n \geq 1$, define

$$
\mathcal{G}_{n}=\left\{f \text { on } S^{n} \mid 0 \leq f \leq 1, U_{n} f=f\right\},
$$


and

$$
\mathscr{H}_{n}=\left\{f \text { on } T_{n} \mid 0 \leq f \leq 1, V_{n} f=f\right\} .
$$

Note that $\mathcal{H}_{1}=g_{1}=\mathcal{H}$, which was defined in $\S 1$.

Lemma 3.10. There is a one-to-one correspondence between $\mathcal{G}_{n}$ and $\mathcal{H}_{n}$ for $n \geq 2$, which is given for $g \in \mathcal{G}_{n}$ and $h \in \mathcal{\alpha}_{n}$ by any one of the following equivalent conditions:

(a) $|g(\vec{x})-h(\vec{x})| \leq n G_{n}(\vec{x})$ for $\vec{x} \in T_{n}$;

(b) $h(\vec{x})=\lim _{k \rightarrow \infty} V_{n}^{k} g(\vec{x})$ for $\vec{x} \in T_{n}$;

(c) $g(\vec{x})=\lim _{k \rightarrow \infty} U_{n}^{k} \tilde{h}(\vec{x})$ for $\vec{x} \in S^{n}$, where $\tilde{h}$ is any extension of $h$ to $S^{n}$ such that $0 \leq \tilde{h}(\vec{x}) \leq 1$.

Proof. Fix $n$, and define $u(\vec{x})=\min \left(n G_{n}(\vec{x}), 2\right)$. We will show first that the three conditions are equivalent. (a) implies (b), since $V_{n}{ }^{k} u(\vec{x}) \rightarrow 0$ for $\vec{x} \in T_{n}$ as $k \rightarrow \infty$ by Corollary 3.7. (a) implies (c) since

$$
|g(\vec{x})-\tilde{h}(\vec{x})| \leq n G_{n}(x)+\sum_{i=1}^{n} \sum_{\substack{j=1 \\ i \neq j}}^{n} p^{(0)}\left(x_{i}, x_{j}\right),
$$

and therefore $U_{n}^{k}(g-\tilde{h})=g-U_{n}^{k} \tilde{h} \rightarrow 0$ as $k \rightarrow \infty$ by Lemma 3.1. On the other hand, (b) implies (a) since

$$
\left|g(\vec{x})-V_{n}^{k} g(\vec{x})\right| \leq n G_{n}(\vec{x})
$$

for $\vec{x} \in T_{n}$ by Lemma 3.8. A similar application of the lemma shows that (c) implies (a). To complete the proof of this lemma, it suffices to show that the limit in (b) exists if $g \in \mathcal{G}_{n}$ and the limit in (c) exists if $h \in \mathcal{H}_{n}$. We will show the first of these only, since the other is similar. If $g \in \mathcal{G}_{n}$, then $U_{n}^{k} g=g$, so

$$
\left|g(\vec{x})-V_{n}{ }^{k} g(\vec{x})\right| \leq u(\vec{x})
$$

for $\vec{x} \in T_{n}$ and $k \geq 1$ by Lemma 3.8. Therefore, for $l \leq k$ and $\vec{x} \in T_{n}$,

$$
\left|V_{n}^{l} g(\vec{x})-V_{n}^{k} g(\vec{x})\right| \leq V_{n}^{l} u(\vec{x}) \rightarrow 0
$$

as $l \rightarrow \infty$ by Corollary 3.7. Hence $\lim _{k \rightarrow \infty} V_{n}{ }^{k} g(\vec{x})$ exists and is in $\alpha_{n}$.

As a consequence of this lemma, the map $\tau_{n}: \mathscr{G}_{n} \rightarrow \mathcal{H}_{n}$ can be defined by $h=\tau_{n}(g)$ for $n \geq 2$. It is natural to define $\tau_{1}$ to be the identity map on 4 . It should be noted that $g \in \mathcal{G}_{n}$ is a symmetric function if and only if $\tau_{n}(g)$ is a symmetric function.

Corollary 3.12. Suppose $g \in \mathcal{G}_{n}, g$ is a symmetric function of its $n$ arguments, and for $\vec{x} \in T_{n}$,

$$
2 g(\vec{x}) \leq g\left(x_{1}, x_{1}, x_{3}, \ldots, x_{n}\right)+g\left(x_{2}, x_{2}, x_{3}, \ldots, x_{n}\right) .
$$


Then $h(\vec{x}) \leq g(\vec{x})$ for $\vec{x} \in T_{n}$, where $h=\tau_{n}(g)$. Furthermore, $g=h$ on $T_{n}$ if and only if equality holds in (3.13) whenever $p\left(x_{1}, x_{2}\right)>0$.

Proof. For $\vec{x} \in T_{n}$,

$$
\begin{aligned}
& g(\vec{x})-V_{n} g(\vec{x})= U_{n} g(\vec{x})-V_{n} g(\vec{x}) \\
&=\frac{1}{n} \sum_{i=1}^{n} \sum_{j=i+1}^{n} p\left(x_{i}, x_{j}\right)\left[g\left(x_{1}, \ldots, x_{i-1}, x_{j}, x_{i+1}, \ldots, x_{n}\right)\right. \\
&\left.\quad+g\left(x_{1}, \ldots, x_{j-1}, x_{i}, x_{j+1}, \ldots, x_{n}\right)-2 g(\vec{x})\right] .
\end{aligned}
$$

So, $V_{n} g \leq g$ on $T_{n}$, and equality holds if and only if equality holds in (3.13) whenever $p\left(x_{1}, x_{2}\right)>0$. Therefore, $V_{n}^{k} g$ is monotonically decreasing in $k$ for $\vec{x} \in T_{n}$. Since $h(\vec{x})=\lim _{k \rightarrow \infty} V_{n}^{k} g(\vec{x})$ on $T_{n}$, the conclusion follows.

The following result, which is of independent interest, characterizes the bounded harmonic functions for the independent finite particle system in terms of those of the one-particle Markov chain.

Lemma 3.14. Suppose $0 \leq g \leq 1$ on $S^{n}$. Then $U_{n} g=g$ if and only if

$$
\sum_{y} p\left(x_{i}, y\right) g\left(x_{1}, \ldots, x_{i-1}, y, x_{i+1}, \ldots, x_{n}\right)=g(\vec{x})
$$

for all $\vec{x} \in S^{n}$ and $1 \leq i \leq n$.

Proof. It follows immediately from the definition of $U_{n}$ that condition (3.15) implies that $U_{n} g=g$. For the converse, note first that $\mathcal{G}_{n}$ can be regarded as a compact, convex subset of $l_{\infty}\left(S^{n}\right)$ with the weak* topology. So, by the Krein-Millman theorem, $\boldsymbol{G}_{n}$ is the closed convex hull of its extreme points. Let $g$ be an extreme point of $\mathcal{G}_{n}$, and define

$$
u_{i}(\vec{x})=\sum_{y} p\left(x_{i}, y\right) g\left(x_{1}, \ldots, x_{i-1}, y, x_{i+1}, \ldots, x_{n}\right)
$$

for $1 \leq i \leq n$. Then $U_{n} u_{i}=u_{i}$ and $0 \leq u_{i} \leq 1$ for each $i$, so $u_{i} \in \mathcal{G}_{n}$. Since $g=U_{n} g=n^{-1}\left(u_{1}+\cdots+u_{n}\right)$ and $g$ is an extreme point of $\mathcal{G}_{n}, g=u_{i}$ for each $i$. Therefore (3.15) holds for each extreme point of $\mathcal{G}_{n}$, and hence for each $g \in \mathcal{G}_{n}$.

It will be convenient in $\S 4$ to use also operators $W_{n}$ which are closely related to $U_{n} . W_{n} f$ is defined for nonnegative functions $f$ on $S^{n}$ by

$$
W_{n} f(\vec{x})=\sum_{i=1}^{n} \sum_{y_{i} \in S} \prod_{j=1}^{n} p\left(x_{j}, y_{j}\right) f(\vec{y}) .
$$

Lemma 3.16. (a) $W_{n}{ }^{k} G_{n} \rightarrow 0$ as $k \rightarrow \infty$.

(b) Take $g \in \mathcal{G}_{n}$ and let $h=\tau(g) \in \mathcal{H}_{n}$. Then $W_{n} g=g$ and $\lim _{k \rightarrow \infty} W_{n}^{k} h=g$.

Proof. (a) follows immediately from

$$
W_{n}^{k} G_{n}(\vec{x})=\frac{1}{n} \sum_{i=1}^{n} \sum_{j=1}^{n} \sum_{i=2 k}^{\infty} p^{(l)}\left(x_{i}, x_{j}\right) .
$$


Now, if $g \in \mathcal{G}_{n}$, then $W_{n} g=g$ by Lemma 3.14. The final statement follows from part (a) of Lemma 3.10 and part (a) of this lemma.

The final preliminary fact about the finite particle system which will be needed is

Lemma 3.17. Suppose fon $T_{n}$ is bounded and satisfies $|f(\vec{x})| \leq G_{n+1}\left(x_{1}, \ldots, x_{n}, y\right)$ for all $\vec{x} \in T_{n}$ and some $y \in S$. Then $V_{n}{ }^{k} f(\vec{x}) \rightarrow 0$ as $k \rightarrow \infty$ for $\vec{x} \in T_{n}$.

Proof. Let $u(\vec{x})=\sum_{i=1}^{n} G\left(x_{i}, y\right)$. Then

$$
G_{n+1}\left(x_{1}, \ldots, x_{n}, y\right)=n G_{n}(\vec{x}) /(n+1)+2 u(\vec{x}) /(n+1) .
$$

By Corollary 3.7, it suffices to show that $V_{n}{ }^{k} u(\vec{x}) \rightarrow 0$ for $\vec{x} \in T_{n}$. To do this, first compute $U_{n}^{k} u(\vec{x})=\sum_{i=1}^{n} u_{k}\left(x_{i}\right)$ where for $x \in S$,

$$
u_{k}(x)=\sum_{j=0}^{k}\left(\begin{array}{l}
k \\
j
\end{array}\right)\left(1-\frac{1}{n}\right)^{k-j}\left(\frac{1}{i} K^{\prime}, \backslash_{i=j}^{j} \prod_{l=j}^{\infty} p^{(l)}(x, y) .\right.
$$

Since $V_{n} v(\vec{x})=U_{n} v(\vec{x})$ for $\vec{x} \in T_{n}$ if $v$ is any function of the form $v(\vec{x})$ $=\sum_{i=1}^{n} w\left(x_{i}\right)$, it follows that $V_{n} U_{n}^{k} u=U_{n}^{k+1} u$ on $T_{n}$. So $U_{n}^{k} u=V_{n}{ }^{k} u$ on $T_{n}$ by (3.9). Therefore $V_{n}{ }^{k} u(\vec{x}) \rightarrow 0$ since $\lim _{k \rightarrow \infty} u_{k}(x)=0$ for each $x \in S$.

4. The characterization. This section is devoted to the proof of Theorem 1.3. It will be assumed throughout that the conditions of that theorem hold. We begin with a slight extension of Corollary 1.2, which is restated using the notation introduced in $\S 3$. Its proof is the same as before.

Lemma 4.1. Let $\mu$ be a probability measure on $X$, and define

$$
f_{n}(\vec{x})=\mu\left\{\eta \mid \eta\left(x_{i}\right)=1 \text { for } 1 \leq i \leq n\right\} \text { for } \vec{x} \in T_{n} .
$$

Then

(a) $\mu \in I$ if and only if $V_{n} f_{n}=f_{n}$ for each $n$, and

(b) if $\lim _{k \rightarrow \infty} V_{n}^{k} f_{n}$ exists for each $n$, then $\nu=\lim _{t \rightarrow \infty} S(t) \mu$ exists, and

$$
\nu\left\{\eta \mid \eta\left(x_{i}\right)=1 \text { for } 1 \leq i \leq n\right\}=\lim _{k \rightarrow \infty} V_{n}{ }^{k} f_{n}(\vec{x}) \text { for } \vec{x} \in T_{n} .
$$

Theorem 4.2. If $\alpha(\cdot) \in$ H, then

(a) $\mu_{\alpha}=\lim _{t \rightarrow \infty} S(t) \nu_{\alpha}$ exists, and therefore $\mu_{\alpha} \in \mathcal{I}$.

(b) $\mu_{\alpha}\{\eta \mid \eta(x)=1\}=\alpha(x)$ for all $x \in S$.

(c) $0 \leq \prod_{i=1}^{n} \alpha\left(x_{i}\right)-\mu_{\alpha}\left\{\eta \mid \eta\left(x_{i}\right)=1\right.$ for $\left.1 \leq i \leq n\right\} \leq n G_{n}(\vec{x})$ for $\vec{x} \in T_{n}$.

(d) $\mu_{\alpha}=\nu_{\alpha}$ if and only if $\alpha$ is constant.

Proof. Define $f_{n}(\vec{x})=\prod_{i=1}^{n} \alpha\left(x_{i}\right)$ for $\vec{x} \in S^{n}$. Then $U_{n} f_{n}=f_{n}$ by Lemma 3.14, so $f_{n} \in \mathcal{G}_{n}$. By Lemma $3.10, \lim _{k \rightarrow \infty} V_{n}{ }^{k} f_{n}$ exists for $\vec{x} \in T_{n}$. So, since

$$
f_{n}(\vec{x})=\nu_{\alpha}\left\{\eta \mid \eta\left(x_{i}\right)=1 \text { for } 1 \leq i \leq n\right\}
$$


for $\vec{x} \in T_{n}, \mu_{\alpha}=\lim _{t \rightarrow \infty} S(t) \nu_{\alpha}$ exists by Lemma 4.1 and

$$
\mu_{\alpha}\left\{\eta \mid \eta\left(x_{i}\right)=1 \text { for } 1 \leq i \leq n\right\}=\lim _{k \rightarrow \infty} V_{n}{ }^{k} f_{n}(\vec{x})
$$

for $\vec{x} \in T_{n} \cdot \mu_{\alpha}\{\eta \mid \eta(x)=1\}=\alpha(x)$ since $V_{1} f_{1}=U_{1} f_{1}=f_{1}$. The second inequality in (c) comes from part (a) of Lemma 3.10. The first inequality is a consequence of Corollary 3.12, since

$$
f_{n}\left(x_{1}, x_{1}, x_{3}, \ldots, x_{n}\right)+f_{n}\left(x_{2}, x_{2}, x_{3}, \ldots, x_{n}\right)-2 f_{n}(\vec{x})=\prod_{i=3}^{n} \alpha\left(x_{i}\right)\left[\alpha\left(x_{1}\right)-\alpha\left(x_{2}\right)\right]^{2},
$$

which is nonnegative. Another consequence is that $\mu_{\alpha}=\nu_{\alpha}$ if and only if $\alpha(x)=\alpha(y)$ for all $x, y$ such that $p(x, y)>0$. Since $p(x, y)$ is irreducible, $\mu_{\alpha}=\nu_{\alpha}$ if and only if $\alpha$ is constant.

The remainder of the results of this section have as their goal the proof that for $\alpha \in \mathcal{H}, \mu_{\alpha}$ is an extreme point of $\mathcal{I}$ and that $\alpha \rightarrow \mu_{\alpha}$ maps onto $I_{e}$. The proof relies heavily on monotonicity considerations.

Lemma 4.3. Take $\mu \in \mathcal{I}$, and define

$$
h_{n}(\vec{x})=\mu\left\{\eta \mid \eta\left(x_{i}\right)=1 \text { for } 1 \leq i \leq n\right\}
$$

for $\vec{x} \in S^{n}$. Then $\lim _{k \rightarrow \infty} U_{n}^{k} h_{n}$ exists, and

$$
h_{n}(\vec{x}) \leq \lim _{k \rightarrow \infty} U_{n}^{k} h_{n}(\vec{x}) \text { for } \vec{x} \in T_{n} .
$$

Proof. The restriction of $h_{n}$ to $T_{n}$ is in $\mathcal{H}_{n}$ by part (a) of Lemma 4.1. Define $g_{n} \in \mathcal{G}_{n}$ by $h_{n}=\tau_{n}\left(g_{n}\right)$. Then $g_{n}(\vec{x})=\lim _{k \rightarrow \infty} U_{n}^{k} h_{n}(\vec{x})$ by Lemma 3.10. So, it remains to be shown that $h_{n}(\vec{x}) \leq g_{n}(\vec{x})$ for $\vec{x} \in T_{n}$, which will follow from Corollary 3.12 if we verify that $g_{n}$ satisfies condition (3.13). By Lemma 3.16, $W_{n}{ }^{k} h_{n} \rightarrow g_{n}$ as $k \rightarrow \infty$, so it suffices to show that $W_{n}{ }^{k} h_{n}$ satisfies (3.13) for each $k$. But

$$
\begin{gathered}
W_{n}{ }^{k} h_{n}\left(x_{1}, x_{1}, x_{3}, \ldots, x_{n}\right)+W_{n}{ }^{k} h_{n}\left(x_{2}, x_{2}, x_{3}, \ldots, x_{n}\right)-2 W_{n}{ }^{k} h_{n}(\vec{x}) \\
=\sum_{\vec{y} \in S^{n}} \prod_{i=1}^{2}\left[p^{(k)}\left(x_{1}, y_{i}\right)-p^{(k)}\left(x_{2}, y_{i}\right)\right] \prod_{j=3}^{n} p^{(k)}\left(x_{j}, y_{j}\right) h_{n}(\vec{y}) \\
=\int_{X}\left\{w_{k}\left(x_{1}, \eta\right)-w_{k}\left(x_{2}, \eta\right)\right\}^{2} \prod_{i=3}^{n} w_{k}\left(x_{i}, \eta\right) \mu(d \eta) \geq 0,
\end{gathered}
$$

since $h_{n}(\vec{y})=\int_{X}\left[\prod_{i=1}^{n} \eta\left(y_{i}\right)\right] \mu(d \eta)$, where $w_{k}(x, \eta)=\sum_{y} p^{(k)}(x, y) \eta(y)$.

Lemma 4.4. Suppose that $\mu \in \mathcal{I}$, and $z \in S$ satisfies $\mu\{\eta \mid \eta(z)=1\}>0$. Define a probability measure $\nu$ on $X$ by $\nu(A)=\mu(A, \eta(z)=1) / \mu\{\eta \mid \eta(z)=1\}$. Then $\tilde{\nu}=\lim _{t \rightarrow \infty} S(t) \nu$ exists, and therefore $\tilde{\nu} \in \mathcal{I}$. Furthermore,

$$
\tilde{\nu}\{\eta \mid \eta(x)=1\} \geq \nu\{\eta \mid \eta(x)=1\}
$$


for $x \neq z$.

Proof. Let $h_{n}(\vec{x})=\mu\left\{\eta \mid \eta\left(x_{i}\right)=1\right.$ for $\left.1 \leq i \leq n\right\}$, and

$$
f_{n}(\vec{x})=\nu\left\{\eta \mid \eta\left(x_{i}\right)=1 \text { for } 1 \leq i \leq n\right\}=h_{n+1}\left(x_{1}, \ldots, x_{n}, z\right) / h_{1}(z) .
$$

Since $\mu \in \mathcal{I}, h_{n} \in \mathcal{H}_{n}$. Define $g_{n} \in \mathcal{G}_{n}$ by $h_{n}=\tau_{n}\left(g_{n}\right)$, and put

$$
\tilde{g}_{n}(\vec{x})=\frac{g_{n+1}\left(x_{1}, \ldots, x_{n}, z\right)}{h_{1}(z)} \text { for } \vec{x} \in S^{n}
$$

Now $g_{n+1} \in \mathcal{G}_{n+1}$ and $0 \leq \tilde{g}_{n} \leq 1$, so $\tilde{g}_{n} \in \mathcal{G}_{n}$ by Lemma 3.14. Therefore $\tau_{n}\left(\tilde{g}_{n}\right)=\lim _{k \rightarrow \infty} V_{n}{ }^{k} \tilde{g}_{n}$ exists by Lemma 3.10. However, by the same lemma,

$$
\begin{aligned}
\left|f_{n}(\vec{x})-\tilde{g}_{n}(\vec{x})\right| & =\left(1 / h_{1}(z)\right)\left|h_{n+1}\left(x_{1}, \ldots, x_{n}, z\right)-g_{n+1}\left(x_{1}, \ldots, x_{n}, z\right)\right| \\
& \leq(n+1) G_{n+1}\left(x_{1}, \ldots, x_{n}, z\right) / h_{1}(z) .
\end{aligned}
$$

By Lemma 3.17, $\lim _{k \rightarrow \infty} V_{n}{ }^{k} f_{n}=\lim _{k \rightarrow \infty} V_{n}^{k} \tilde{g}_{n}=\tau_{n}\left(\tilde{g}_{n}\right)$ on $T_{n}$. So, $\tilde{v}$ $=\lim _{t \rightarrow \infty} S(t) \nu$ exists by Lemma 4.1, and

$$
\tilde{\nu}\left\{\eta \mid \eta\left(x_{i}\right)=1 \text { for } 1 \leq i \leq n\right\}=\tau_{n}\left(\tilde{g}_{n}\right)(\vec{x})
$$

for $\vec{x} \in T_{n}$. To obtain the inequality, note that, for $x \in S$,

$$
\tilde{\nu}\{\eta \mid \eta(x)=1\}=\tau_{1}\left(\tilde{g}_{1}\right)(x)=\tilde{g}_{1}(x)=g_{2}(x, z) / h_{1}(z),
$$

and

$$
\nu\{\eta \mid \eta(x)=1\}=h_{2}(x, z) / h_{1}(z) .
$$

But $h_{2}(x, z) \leq g_{2}(x, z)$ for $x \neq z$ by Lemma 4.3.

The following basic theorem is a direct consequence of this result.

Theorem 4.5. If $\mu \in I_{e}$, then for $x \neq z$,

$$
\mu\{\eta \mid \eta(x)=1, \eta(z)=1\} \leq \mu\{\eta \mid \eta(x)=1\} \mu\{\eta \mid \eta(z)=1\} .
$$

Proof. If $\mu\{\eta \mid \eta(x)=0$ for all $x\}=1$ or $\mu\{\eta \mid \eta(x)=1$ for all $x\}=1$, then the result is obvious. In all other cases, $0<\mu\{\eta \mid \eta(x)=1\}<1$ for all $x \in S$, since $\mu\{\eta \mid \eta(x)=1\} \in \mathcal{\&}$ by Lemma 4.1. Fix $z \in S$, and define probability measures $\nu_{1}$ and $\nu_{2}$ on $X$ by $\nu_{1}(A)=\mu(A, \eta(z)=1) / \mu\{\eta \mid \eta(z)=1\}, \nu_{2}(A)=\mu(A, \eta(z)=0)$ $/ \mu\{\eta \mid \eta(z)=0\}$. Put $\lambda=\mu\{\eta \mid \eta(z)=1\}$. Then $\mu=\lambda \nu_{1}+(1-\lambda) \nu_{2}$, so

$$
\mu=S(t) \mu=\lambda S(t) \nu_{1}+(1-\lambda) S(t) \nu_{2} .
$$

By Lemma 4.4, $\tilde{\nu}_{1}=\lim _{t \rightarrow \infty} S(t) \nu_{1}$ exists. Therefore $\tilde{\nu}_{2}=\lim _{t \rightarrow \infty} S(t) \nu_{2}$ exists also, and $\mu=\lambda \tilde{\nu}_{1}+(1-\lambda) \tilde{\nu}_{2}$. Since $\tilde{\nu}_{1}$ and $\tilde{\nu}_{2} \in \mathcal{I}$, and $\mu \in \mathcal{I}_{e}$, it follows that $\mu=\tilde{\nu}_{1}=\tilde{\nu}_{2}$. By the second part of Lemma 4.4, 


$$
\begin{aligned}
\mu\{\eta \mid \eta(x)=1\} & =\tilde{\nu}_{1}\{\eta \mid \eta(x)=1\} \geq \nu_{1}\{\eta \mid \eta(x)=1\} \\
& =\frac{\mu\{\eta \mid \eta(x)=1, \eta(z)=1\}}{\mu\{\eta \mid \eta(z)=1\}}
\end{aligned}
$$

for $x \neq z$, which completes the proof.

Remark. For $\alpha \in \mathcal{H}, \mu_{\alpha}$ satisfies (4.6) by part (c) of Theorem 4.2. So, Theorem 4.5 would have been immediate if we knew at this point that $I_{e}=\left\{\mu_{\alpha} \mid \alpha \in \mathcal{L}\right\}$. However, this theorem plays a crucial role in proving this fact.

Lemma 4.7. Suppose $\mu$ is a probability measure on $X$ and define $f(x, y)$ $=\mu\{\eta \mid \eta(x)=1, \eta(y)=1\}$ for $x, y \in S$. If

$$
f(x, y) \leq f(x, x) f(y, y) \quad \text { for } x \neq y
$$

then

$$
\begin{aligned}
\operatorname{var}\left[\sum_{x} \eta(x) q(x)\right] & =\int_{X}\left\{\sum_{x}[\eta(x) q(x)-f(x, x) q(x)]\right\}^{2} d \mu \\
& \leq \sum_{x}[q(x)]^{2}
\end{aligned}
$$

whenever $q(x) \geq 0$ and $\sum_{x} q(x)<\infty$.

Proof.

$$
\begin{aligned}
\operatorname{var}\left[\sum_{x} \eta(x) q(x)\right] & =\int\left[\sum_{x} \eta(x) q(x)\right]^{2} d \mu-\left[\sum_{x} f(x, x) q(x)\right]^{2} \\
& =\sum_{x, y} f(x, y) q(x) q(y)-\sum_{x, y} f(x, x) f(y, y) q(x) q(y) \\
& \leq \sum_{x}[q(x)]^{2} f(x, x)[1-f(x, x)] \leq \sum_{x}[q(x)]^{2},
\end{aligned}
$$

where the next to the last inequality follows from (4.8).

Corollary 4.9. In addition to the assumptions of Lemma 4.7, suppose that $q_{i}(x)$ satisfies $q_{i}(x) \geq 0$ and $\sum_{x} q_{i}(x) \leq 1$ for $1 \leq i \leq n$. Then

$$
\left\{\int_{X} \prod_{i=1}^{n}\left[\sum_{x}\left(\eta(x) q_{i}(x)-f(x, x) q_{i}(x)\right)\right] d \mu\right\}^{2} \leq \min _{1 \leq i \leq n}\left[\sum_{x} q_{i}^{2}(x)\right] .
$$

Proof. Since $\left|\sum_{x}\left(\eta(x) q_{i}(x)-f(x, x) q_{i}(x)\right)\right| \leq 1$ for each $i$, the result follows from Lemma 4.7 by applying Hölder's inequality.

Theorem 4.10. Suppose $\mu \in \mathcal{I}$, and define $\alpha \in$ \& by $\alpha(x)=\mu\{\eta \mid \eta(x)=1\}$. If $\mu$ satisfies

$$
\mu\{\eta \mid \eta(x)=1, \eta(y)=1\} \leq \alpha(x) \alpha(y)
$$

for $x \neq y$, then $\mu=\mu_{\alpha}$. 
Proof. That the $\alpha$ so defined is in $\alpha$ follows from part (a) of Lemma 4.1. Define

$$
f_{n}\left(x_{1}, \ldots, x_{n}\right)=\mu\left\{\eta \mid \eta\left(x_{i}\right)=1 \text { for } 1 \leq i \leq n\right\}
$$

and

$$
h_{n}\left(x_{1}, \ldots, x_{n}\right)=\mu_{\alpha}\left\{\eta \mid \eta\left(x_{i}\right)=1 \text { for } 1 \leq i \leq n\right\}
$$

If suffices to show that $f_{n}=h_{n}$ for each $n \geq 1$. The proof is by induction on $n$. The case $n=1$ is an immediate consequence of part (b) of Theorem 4.2 and the definition of $\alpha$. Assume now that $f_{n-1}=h_{n-1}$. Then

$$
\begin{aligned}
W_{n}{ }^{k} f_{n}(\vec{x})-W_{n}{ }^{k} h_{n}(\vec{x})= & \sum_{\vec{y} \in S^{n}} \prod_{j=1}^{n} p^{(k)}\left(x_{j}, y_{j}\right)\left[f_{n}(\vec{y})-h_{n}(\vec{y})\right] \\
= & \int_{X} \prod_{i=1}^{n}\left[\sum_{y \in S}(\eta(y)-\alpha(y)) p^{(k)}\left(x_{i}, y\right)\right] d \mu \\
& -\int_{X} \prod_{i=1}^{n}\left[\sum_{y \in S}(\eta(y)-\alpha(y)) p^{(k)}\left(x_{i}, y\right)\right] d \mu_{\alpha}
\end{aligned}
$$

where the first equality comes from the definition of $W_{n}$, while the second comes from the induction hypothesis. Therefore, by Corollary 4.9, since both $\mu$ and $\mu_{\alpha}$ satisfy the assumptions of Lemma 4.7 ,

$$
\begin{aligned}
\left|W_{n}{ }^{k} f_{n}(\vec{x})-W_{n}{ }^{k} h_{n}(\vec{x})\right| & \leq 2 \min _{1 \leq i \leq n}\left(\sum_{y}\left[p^{(k)}\left(x_{i}, y\right)\right]^{2}\right)^{1 / 2} \\
& =2 \min _{1 \leq i \leq n}\left(p^{(2 k)}\left(x_{i}, x_{i}\right)\right)^{1 / 2}
\end{aligned}
$$

But this term tends to zero as $k \rightarrow \infty$ for each $\vec{x} \in S^{n}$ since $p$ is transient. Since $f_{n}, h_{n} \in \mathcal{H}_{n}$ by Lemma 4.1, $g_{n}, \tilde{g}_{n} \in \mathcal{G}_{n}$ can be defined by $f_{n}=\tau_{n}\left(g_{n}\right)$ and $h_{n}=\tau_{n}\left(\tilde{g}_{n}\right)$. Since $W_{n}{ }^{k} f_{n}-W_{n}{ }^{k} h_{n} \rightarrow 0$ as $k \rightarrow \infty$, Lemma 3.16 gives that $g_{n}$ $=\tilde{g}_{n}$. Since $\tau_{n}$ is one-to-one, $f_{n}=h_{n}$ and the induction step is complete.

Theorems 4.5 and 4.10 now yield

Corollary 4.11. $I_{e} \subset\left\{\mu_{\alpha} \mid \alpha \in \mathcal{H}\right\}$. Therefore, $I=$ closed convex hull of $\left\{\mu_{\alpha} \mid \alpha \in \mathcal{H}\right\}$.

To complete the proof of Theorem 1.3, it remains to be shown that for each $\alpha \in \mathcal{H}, \mu_{\alpha} \in I_{e}$. In order to do this, it is convenient to give $\& \subset l_{\infty}(S)$ the topology it inherits from the weak* topology on $l_{\infty}(S)$. With this topology, $\&$ is compact. Let $C(\&)$ be the space of continuous functions on $\&$ with the supremum norm.

Lemma 4.12. Let $\exists$ be the collection of all functions on $\mathcal{A}$ of the form $f(\alpha)=\prod_{i=1}^{n} \alpha\left(x_{i}\right)$, where $n \geq 1$ and $x_{i} \in S$ are arbitrary, and in addition the constant function 1. Then the linear span of $\exists$ is dense in $C(\alpha)$. 
Proof. The linear span of $\exists$ is an algebra in $C(4)$ which separates points and contains the constants. So, the result follows from the Stone-Weierstrass theorem.

Lemma 4.13. Let $\tilde{\Im}$ be the collection of all functions on 4 of the form $f(\alpha)=\int_{X} \eta\left(x_{1}\right) \ldots \eta\left(x_{n}\right) d \mu_{\alpha}$, where $n \geq 1$ and $x_{i} \in S$ are arbitrary, and in addition the constant function 1 . Then the linear span of $\tilde{\mathcal{G}}$ is dense in $C(\mathcal{H})$.

Proof. For $\vec{x} \in S^{n}$ and $\alpha \in \mathcal{H}$, define

$$
g(\vec{x} ; \alpha)=\prod_{i=1}^{n} \alpha\left(x_{i}\right) \quad \text { and } \quad h(\vec{x} ; \alpha)=\int_{X} \eta\left(x_{1}\right) \ldots \eta\left(x_{n}\right) d \mu_{\alpha} .
$$

Then for each $\alpha, h=\tau_{n}(g)$ by Lemma 4.1 and Theorem 4.2. Therefore,

$$
h(\vec{x} ; \alpha)=\lim _{k \rightarrow \infty} V_{n}^{k} g(\vec{x} ; \alpha) \quad \text { for } \vec{x} \in T_{n},
$$

and

$$
g(\vec{x} ; \alpha)=\lim _{k \rightarrow \infty} U_{n}^{k} h(\vec{x} ; \alpha) \quad \text { for } \vec{x} \in S^{n}
$$

by Lemma 3.10. An examination of the proof of that lemma shows that the convergence is uniform in $\alpha$. Since $g(\vec{x} ; \alpha)$ is continuous in $\alpha$, it follows from (4.14) that $h(\vec{x} ; \alpha)$ is also. But $U_{n}^{k} h(\vec{x} ; \alpha)$ is in the closure of the linear span of $\tilde{\mathcal{G}}$, and therefore $g$ is also by (4.15). The desired conclusion then follows from Lemma 4.12.

Theorem 4.16. For each $\alpha \in \mathcal{H}, \mu_{\alpha} \in I_{e}$.

Proof. By Corollary 4.11 and Choquet's theorem, it suffices to show that if

$$
\mu_{\alpha}=\int_{d} \mu_{\beta} \nu(d \beta)
$$

where $\nu$ is a probability measure on $\mathcal{H}$, then $\nu$ is the point mass at $\alpha$. From (4.17), it follows that

$$
f(\alpha)=\int_{\alpha} f(\beta) \nu(d \beta)
$$

for all $f \in \tilde{\mathcal{G}}$. So, (4.18) holds for all $f \in C(\mathcal{A})$ by Lemma 4.13. Therefore, $\nu$ is the point mass at $\alpha$.

5. Ergodic theorems. This section is devoted to the proof of Theorem 1.5 and several of its consequences. The assumptions of Theorem 1.3 are to hold throughout. It is convenient to introduce the continuous time versions of the operators $U_{n}$ and $V_{n}$ :

$$
U_{n}^{t} f(\vec{x})=e^{-t} \sum_{k=0}^{\infty} \frac{t^{k}}{k !} U_{n}^{k} f(\vec{x})
$$


and

$$
V_{n}^{t} f(\vec{x})=e^{-t} \sum_{k=0}^{\infty} \frac{t^{k}}{k !} V_{n}^{k} f(\vec{x})
$$

Lemma 5.1. Suppose $0 \leq f(\vec{x}) \leq 1$ for $\vec{x} \in S^{n}, g \in \mathcal{G}_{n}$, and $h=\tau_{n}(g) \in \mathcal{\alpha}_{n}$. Then

$$
\lim _{t \rightarrow \infty} V_{n}^{t} f(\vec{x})=h(\vec{x}) \text { for all } \vec{x} \in T_{n}
$$

if and only if $\lim _{t \rightarrow \infty} U_{n}^{t} f(\vec{x})=g(\vec{x})$ for all $\vec{x} \in S^{n}$.

Proof. Suppose $\lim _{t \rightarrow \infty} U_{n}^{t} f=g$ on $S^{n}$. Then for $\vec{x} \in T_{n}$,

$$
\begin{aligned}
\left|V_{n}^{t} f(\vec{x})-h(\vec{x})\right| & \leq\left|V_{n}^{t} f(\vec{x})-U_{n}^{t} f(\vec{x})\right|+\left|U_{n}^{t} f(\vec{x})-g(\vec{x})\right|+|g(\vec{x})-h(\vec{x})| \\
& \leq 2 n G_{n}(\vec{x})+\left|U_{n}^{t} f(\vec{x})-g(\vec{x})\right|
\end{aligned}
$$

by Lemmas 3.8 and 3.10. Therefore, $\lim \sup _{t \rightarrow \infty}\left|V_{n}^{t} f(\vec{x})-h(\vec{x})\right| \leq 2 n u(\vec{x})$ where $u(\vec{x})=\min \left(G_{n}(\vec{x}), 1\right)$. Since $h \in \mathcal{H}_{n}, V_{n}^{t} h=h$. Using $V_{n}^{t+s}=V_{n}^{s} V_{n}^{t}$, it then follows that

$$
\begin{aligned}
\limsup _{t \rightarrow \infty}\left|V_{n}^{t} f(\vec{x})-h(\vec{x})\right| & =\limsup _{t \rightarrow \infty}\left|V_{n}^{t}(f-h)(\vec{x})\right| \\
& \leq V_{n}^{s}\left[\limsup _{t \rightarrow \infty}\left|V_{n}^{t}(f-h)(\vec{x})\right|\right] \\
& \leq 2 n V_{n}^{s} u(\vec{x}) .
\end{aligned}
$$

By Corollary 3.7, $\lim _{s \rightarrow \infty} V_{n}^{s} u(\vec{x})=0$, so $\lim _{t \rightarrow \infty} V_{n}^{t} f(\vec{x})=h(\vec{x})$. The converse is proved in the same way, using Lemma 3.1 instead of Corollary 3.7.

Proof of Theorem 1.5. Let $\mu$ be a probability measure on $X$ and $\alpha \in \mathcal{H}$. Define

$$
\begin{array}{ll}
g_{n}(\vec{x})=\prod_{i=1}^{n} \alpha\left(x_{i}\right) & \text { for } \vec{x} \in S^{n}, \\
h_{n}(\vec{x})=\mu_{\alpha}\left\{\eta \mid \eta\left(x_{i}\right)=1 \text { for } 1 \leq i \leq n\right\} & \text { for } \vec{x} \in T_{n},
\end{array}
$$

and

$$
f_{n}(\vec{x})=\mu\left\{\eta \mid \eta\left(x_{i}\right)=1 \text { for } 1 \leq i \leq n\right\} \text { for } \vec{x} \in S^{n}
$$

From the proof of Corollary 1.2, it is easily seen that $\lim _{t \rightarrow \infty} S(t) \mu=\mu_{\alpha}$ if and only if

$$
\lim _{t \rightarrow \infty} V_{n}^{t} f_{n}(\vec{x})=h_{n}(\vec{x})
$$


for each $\vec{x} \in T_{n}$ and $n \geq 1$. Since $h_{n}=\tau_{n}\left(g_{n}\right)$, Lemma 5.1 shows that (5.2) holds if and only if

$$
\lim _{t \rightarrow \infty} U_{n}^{t} f_{n}(\vec{x})=g_{n}(\vec{x})
$$

for each $\vec{x} \in S^{n}$ and $n \geq 1$. However,

$$
U_{n}^{n t} f_{n}(\vec{x})=\sum_{i=1}^{n} \sum_{y_{i} \in S} \prod_{j=1}^{n} p_{t}\left(x_{j}, y_{j}\right) f_{n}(\vec{y}) .
$$

So, applying (5.3) for $n=1$ and $n=2$ yields

$$
\lim _{t \rightarrow \infty} \sum_{y} p_{t}(x, y) f_{1}(y)=\alpha(x),
$$

and

$$
\lim _{t \rightarrow \infty} \sum_{y, z} p_{t}(x, y) p_{t}(x, z) f_{2}(y, z)=[\alpha(x)]^{2},
$$

which says that

$$
\int_{X}\left\{\sum_{y} p_{t}(x, y)[\eta(y)-\alpha(y)]\right\}^{2} d \mu \rightarrow 0
$$

as $t \rightarrow \infty$ and hence that (1.6) holds. Conversely, if (1.6) holds, then

$$
\int_{X} \prod_{i=1}^{n}\left\{\sum_{y} p_{t}\left(x_{i}, y\right)[\eta(y)-\alpha(y)]\right\} d \mu \rightarrow 0
$$

as $t \rightarrow \infty$ for each $n \geq 1$ and $\vec{x} \in S^{n}$. The proof that (5.3) holds is then by induction on $n$, and is omitted since it is quite similar to the proof of Theorem 4.10.

The following simple consequence shows that if $\mu$ satisfies

$$
\alpha(x) \equiv \mu\{\eta \mid \eta(x)=1\} \in \text { \& }
$$

and $\mu$ is mixing in an appropriate sense, then $S(t) \mu \rightarrow \mu_{\alpha}$ as $t \rightarrow \infty$. In order to define mixing, it is necessary to have some concept of distance in $S$. This is done in terms of the function $G(x, y)$, the idea being that $x$ and $y$ are far apart if $G(x, y)$ is small.

Corollary 5.5. Suppose $\mu$ is a probability measure on $X$ and

$$
\alpha(x) \equiv \mu\{\eta \mid \eta(x)=1\} \in \mathcal{H}
$$

Put $f(x, y)=\mu\{\eta \mid \eta(x)=1, \eta(y)=1\}$. If

$$
\lim _{G(x, y) \rightarrow 0 ; x \neq y}|f(x, y)-\alpha(x) \alpha(y)|=0,
$$

then $S(t) \mu \rightarrow \mu_{\alpha}$ as $t \rightarrow \infty$. 
Proof. In order to apply Theorem 1.5, it suffices to show that (5.4) holds, since it is equivalent to (1.6). Given $\varepsilon>0$, choose $\delta>0$ so that $|f(x, y)-\alpha(x) \alpha(y)|$ $<\varepsilon$ whenever $x \neq y$ and $G(x, y)<\delta$. Then

$$
\begin{aligned}
\int_{X}\left\{\sum_{y} p_{t}(x, y)[\eta(y)-\alpha(y)]\right\}^{2} d \mu \\
\quad=\sum_{y, z} p_{t}(x, y) p_{t}(x, z)[f(y, z)-\alpha(y) \alpha(z)] \\
\quad \leq \varepsilon+p_{2 t}(x, x)+\delta^{-1} \sum_{y, z} p_{t}(x, y) p_{t}(x, z) G(y, z) .
\end{aligned}
$$

The proof is complete, since the second two terms tend to zero as $t \rightarrow \infty$, and $\varepsilon$ is arbitrary.

The final application of Theorem 1.5 is

Theorem 5.6. Suppose $S$ is an Abelian group, $p(x, y)=p(0, y-x)$, and $\mu$ is a stationary ergodic probability measure on $X$. Let $\alpha$ be the constant $\mu\{\eta \mid \eta(x)=1\}$. Then $\lim _{t \rightarrow \infty} S(t) \mu=\mu_{\alpha}$.

Proof. Let $\Gamma$ be the group dual to $S$, and $\nu$ be the spectral measure of the process $\eta(x)$ determined by $\mu$ :

$$
\mu\{\eta: \eta(x)=1, \eta(y)=1\}-\alpha^{2}=\int_{\Gamma} \gamma(x-y) \nu(d \gamma) .
$$

Define a function $h$ on $\Gamma$ by $h(\gamma)=\sum_{x \in S} p(0, x) \gamma(x)$. Then $|h(\gamma)| \leq 1$, and $h(\gamma)=1$ if and only if $\gamma=1$ since $p$ is irreducible. Note that $\sum_{y} p_{t}(x, y) \gamma(y)$ $=\gamma(x) \exp \{-t[1-h(\gamma)]\}$. Therefore, putting $Z_{t}(x, \eta)=\sum_{y} p_{t}(x, y) \eta(y)$, it follows that

$$
\begin{aligned}
& \int_{X}\left[Z_{t}(x, \eta)-Z_{s}(x, \eta)\right]^{2} \mu(d \eta) \\
&=\int_{\Gamma}\{\exp [-2 t(1-h(\gamma))]+\exp [-2 s(1-h(\gamma))] \\
&-2 \exp [-(s+t)(1-h(\gamma))]\} \nu(d \gamma)
\end{aligned}
$$

which tends to zero as $t$ and $s$ tend to $\infty$. Therefore,

$$
Z(x, \eta)=\lim _{t \rightarrow \infty} Z_{t}(x, \eta)
$$

exists in mean square. Since by definition

$$
Z_{t+s}(x, \eta)=\sum_{y} p_{s}(x, y) Z_{t}(y, \eta)
$$

it follows that $Z(x, \eta) \in \mathcal{A}$ for a.e. $\eta(\mu)$. Since $p$ is translation invariant, \& consists only of constants. Therefore $Z(x, \eta)=Z(0, \eta)$ a.s. $(\mu)$. This says that $Z(x, \eta)$ is an invariant random variable, so since $\mu$ is ergodic, it follows that 
$Z(x, \eta)$ is constant in $\eta$ a.s. $(\mu)$. Since $\int Z(x, \eta) \mu(d \eta)=\alpha$, we conclude that $Z(x, \eta)=\alpha$ a.s. $(\mu)$ for each $x \in S$. The result then follows from Theorem 1.5, since $\lim _{t \rightarrow \infty} Z_{t}(x, \eta)=\alpha$ in mean square.

\section{REFERENCES}

1. G. Choquet and J. Deny, Sur l'equation de convolution $\mu=\mu * \sigma$, C.R. Acad. Sci. Paris 250 (1960), 799-801. MR 22 \#9808.

2. W. Feller, An introduction to probability theory and its applications, Vol. II, Wiley, New York, 1966. MR 35 \# 1048.

3. R. Holley, Free energy in a Markovian model of a lattice spin system, Comm. Math. Phys. 23 (1971), 87-99.

4. - Pressure and Helmholtz free energy in a dynamic model of a lattice gas, Proc. Sixth Berkeley Sympos. Vol. III,Univ. of Berkeley Press, Berkeley, Calif., 1972, pp. 565-578.

5.—, An ergodic theorem for interacting systems with attractive interactions, $\mathrm{Z}$. Wahrscheinlichkeitstheorie und Verw. Gebiete 24 (1972), 325-334.

6. T.M. Liggett, Existence theorems for infinite particle systems, Trans. Amer. Math. Soc. 165 (1972), 471-481.

7.—, An infinite particle system with zero range interactions, Ann. Probability (to appear).

8. F. Spitzer, Interaction of Markov processes, Advances in Math. 5 (1970), 246-290. MR 42 \# 3856.

Department of Mathematics, University of California, los Angeles, California 90024 